\title{
Predictors of joint damage in patients with rheu- matoid arthritis: focus on short- and long-term effects of intra-articular glucocorticoid injections
}

\author{
Jung Hee Koh ${ }^{1}$, Hanna Lee ${ }^{2}$, and Seung-Geun Lee ${ }^{2}$
}

${ }^{1}$ Division of Rheumatology, Department of Internal Medicine, Bucheon St. Mary's hospital, College of Medicine, The Catholic University of Korea, Seoul; ${ }^{2}$ Division of Rheumatology, Department of Internal Medicine, Pusan National University Hospital, Pusan, Korea

Received: May 1, 2020

Revised : June 26, 2020

Accepted: June 30, 2020

\section{Correspondence to} Jung Hee Koh, M.D.

Division of Rheumatology,

Department of Internal

Medicine, Bucheon St. Mary's

Hospital, College of Medicine,

The Catholic University of Korea,

327 Sosaro, Wonmi-gu, Bucheon

14647, Korea

Tel: $+82-32-340-7728$

Fax: +82-32-340-7227

E-mail:jungheekoh@gmail.com https://orcid.org/0000-0002-

6617-1449
Background/Aims: To investigate the short- and long-term efficacy of intra-articular glucocorticoid injections (IAGI) in patients with rheumatoid arthritis (RA). Methods: This was a retrospective study of RA patients who had active arthritis in the hand or wrist joints and who received IAGI (or not) as an adjunct to disease-modifying antirheumatic drugs (DMARDs). Short-term efficacy was assessed based on changes in the disease activity score in 28 joints (DAS28) after 3 months and long-term efficacy was assessed based on changes in the van der Heijde Sharp score (HSS) of hand radiographs over 2 years. Radiographic progression was defined as $\Delta$ HSS/year $\geq 2$. Logistic regression analysis identified predictors of early achievement of low disease activity (LDA) and radiographic progression.

Results: Overall, 126 RA patients received IAGI into the hand or wrist joints and 107 were IAGI-naive. After 3 months, $67 \%$ of IAGI-treated patients and $48 \%$ of IAGI-naive patients achieved LDA $(p=0.002)$. Over the next 2 years, $35 \%$ of patients treated with IAGI showed radiographic progression compared with $27 \%$ of IAGI-naive patients $(p=0.2)$. IAGI plus biologic DMARDs was associated with achievement of LDA in 3 months. Achieving LDA in 3 months (odds ratio [OR], 0.403; 95\% confidence interval [CI], 0.192 to 0.847 ), wrist arthritis (OR, 2.408; 95\% CI, 1.184 to 4.897 ), and baseline HSS (OR, 1.021; $95 \%$ CI, 1.003 to 1.039) were associated with radiographic progression.

Conclusions: IAGI was associated with early achievement of LDA. LDA was associated with slower radiographic progression. The wrist is more vulnerable to joint damage and requires more aggressive treatment.

Keywords: Rheumatoid arthritis; Intra-articular injection; Glucocorticoids; Disease progression; Wrist

\section{INTRODUCTION}

The goal of modern medical treatments for rheumatoid arthritis (RA) is to achieve remission or low disease activity (LDA) [1]. Intra-articular glucocorticoid injection (IAGI) is used as part of a treat-to-target strategy in these patients [2-4].
Theoretically, IAGI results in rapid control of arthritis by delivering a high concentration of glucocorticoids to the site of inflammation. In those with inflammatory arthritis, intra-articular glucocorticoids reduce the number of synovial $\mathrm{T}$ cells and reduce expression of receptor activator of nuclear factor kappa-B ligands [5]. Previous studies show that IAGI results in effective inflammatory 
control in the injected joints and prevents development of erosive disease [2,6-8].

However, recent studies show that repeated IAGI into the knees of patients with osteoarthritis results in a significant increase in loss of cartilage volume after 2 years $[9,10]$. Another study of patients with early RA shows that IAGI prevents bone loss in the hands during the first 3 months, but has an insignificant effect from the 3 rd to 12th month [3]. Moreover, ultrasound or magnetic resonance imaging conducted by Boesen et al. [11] found no changes in synovitis at 4 weeks post-injection of glucocorticoids. These results, taken together with other in vitro and in vivo results showing gross cartilage damage [12], suggest that IAGI is chondrotoxic. This raises questions about whether IAGI promotes damage in injected joints over the long-term.

There are limited data regarding radiographic changes in the joints of those with RA receiving IAGI. Therefore, the objective of the present study was to investigate whether IAGI has an additional benefit over that of disease-modifying antirheumatic drug (DMARD) treatment alone with respect to 1 ) achievement of LDA after 3 months and 2) limiting radiographic progression over the subsequent 2 years. In addition, we identified factors associated with early achievement of LDA and with radiographic progression.

\section{METHODS}

\section{Study population}

This was a retrospective cohort study of outpatients attending the Rheumatology Department of Pusan National University Hospital (PNUH), a tertiary referral hospital in South Korea. Patient electronic medical records were searched to identify all individuals with RA who received IAGI between January 2011 and December 2015. All patients included in the study met the 2010 RA classification criteria [13]. Informed consent was waived due to the retrospective nature of the study. All aspects of this study were approved by the PNUH Institutional Review Board (IRB no. 1901-014-075).

To compare radiographic changes, all patients underwent a plain radiographic scan of the hands before and after active arthritis. Hand plain radiographs before active arthritis were taken within 1 year before active ar- thritis occurred, whereas hand plain radiographs after active arthritis were taken 1 to 3 years after active arthritis occurred. When the patient received IAGI, follow-up hand radiographs taken at least 1 year after IAGI were used for analysis.

To determine whether IAGI was associated with structural damage, age- and sex-matched RA patients who had active arthritis but never received IAGI were identified and matched to each patient who received IAGI during the same period (Fig. 1). Active arthritis was defined as swollen joints (as documented by rheumatologists).

The 5-year study included 248 patients with RA (81\% females) who received IAGI and 248 age- and sexmatched patients who had active arthritis but never received IAGI (IAGI-naïve). Of these, 286 patients (58\%) had active arthritis in the wrist or hand joints. Paired hand plain radiographs before and after active arthritis were available for 126 patients treated with IAGI and for 107 patients not treated with IAGI (Fig. 1).

The study examined the hand and wrist joints because these joints are not weight-bearing and are relatively free from crystal-induced arthritis. The wrist or hand joints in question included the radiocarpal joint, intercarpal joint, distal radioulnar joint, the metacarpophalangeal (MCP) joints ( 1 to 5 ), the proximal interphalangeal (PIP) joints (2 to 5 ), and the first interphalangeal joint. The radiocarpal, intercarpal, and distal radioulnar joints were grouped as the wrist joint. The first interphalangeal joint was regarded as a PIP joint.

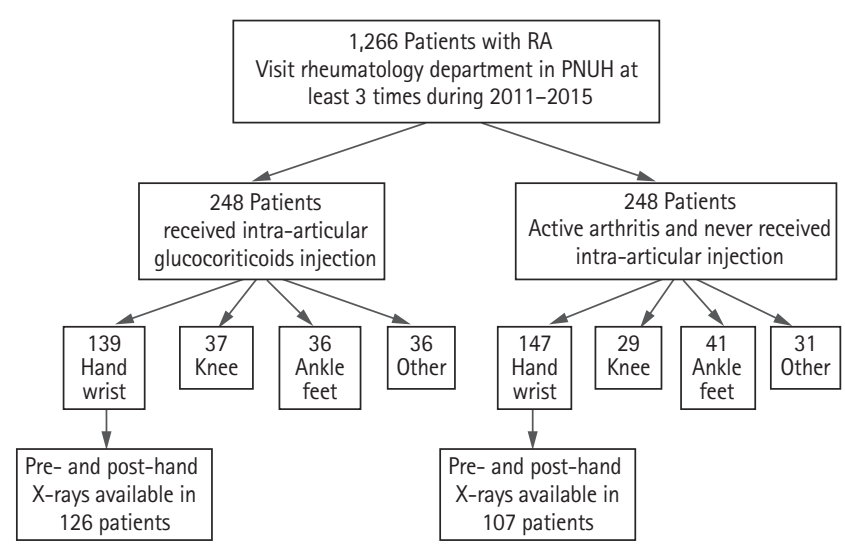

Figure 1. Flow chart showing selection of patients with RA with active disease in the wrist or hand joints. RA, rheumatoid arthritis; PNUH, Pusan National University Hospital. 


\section{Data collection}

Clinical data were assessed at the visit when the patient was diagnosed with active arthritis in the wrist or hand joint. Demographic data and therapeutic strategies (oral glucocorticoids; all disease-modifying antirheumatic drug [DMARDs] prescriptions-including conventional synthetic DMARDs [methotrexate, leflunomide, sulfasalazine, and hydroxychloroquine] and biologic DMARDs [infliximab, etanercept, adalimumab, tocilizumab, abatacept, and rituximab]; and non-steroidal anti-inflammatory drugs [NSAIDs]) given after the patients experienced active arthritis were collected. Treatment decisions were not protocol-based and patients received the usual care from a rheumatologist. Oral glucocorticoids data were converted to daily prednisolone-equivalent dosages.

Serological data, including anti-citrullinated protein antibody (ACPA) and rheumatoid factor (RF) titers were recorded. The level of acute-phase reactants, including C-reactive protein (CRP) and the erythrocyte sediment rate, and the disease activity score in 28 joints (DAS28; at the time of active arthritis and after a follow-up of 3 months after active arthritis), were also recorded.

Radiographic data were collected and evaluated in chronological order following multi-reader (K.J.H. and L.H.) assessment blinded to clinical data. Hand plain radiographs were assessed and quantified according to the modified van der Heijde Sharp score (HSS) based on observation of typical RA erosions and joint space narrowing (JSN) [14]. To estimate inter-observer and intra-observer reliability, 100 randomly selected films were interpreted twice by each reader at an interval of 4 weeks. The Cronbach's $\alpha$ value for the two readers was o.88. The inter-observer correlation coefficient varied from 0.82 to 0.92 . The intraclass correlation coefficients for each reader were 0.89 ( 0.84 to 0.92 ) and 0.91 ( 0.87 to o.94), respectively.

\section{Outcome definitions}

The DAS28 at 3 months post-active arthritis was considered as a short-term outcome. The DAS28 was categorized as remission, or as high, moderate, or LDA, based on American College of Rheumatology recommendations [15]. Treatment response was assessed according to the proportion of patients that achieved remission or LDA. In addition, whether IAGI reduced swollen joint was reviewed by the attending rheumatologist at the follow-up visit.

To investigate long-term outcomes, the estimated yearly rate of overall radiographic progression was calculated by dividing the difference in the hand HSS by the number of years that had elapsed between the "before" and "after" active arthritis hand radiographs. A clinically significant change in the total HSS, which includes hands and feet, is 5 [16]. As the present study assessed hands only, a change in the hand HSS scores of 3 was regarded as clinically significant. Hand radiographs were examined on average at 2 (interquartile range [IQR], 2 to 3) year intervals. Overall hand radiographic progression per year was defined as $\Delta$ HSS/year $\geq 2$.

Because the HSS can be higher in patients with longstanding RA than in those with early RA, the outcome for those with early and established RA was analyzed separately. In this study, early RA was defined as RA with disease duration of $<1$ year, where duration is defined as the length of time the patient had been diagnosed with RA. In addition, no patients with early RA should have radiographic joint damage. Established RA was defined as RA with disease duration of $\geq 1$ year; this also included patients diagnosed with RA who already had radiographic joint damage. In this study, early RA accounted for $29 \%$ of cases and established RA for $71 \%$.

\section{Ultrasound evaluation}

All injections were carried out using ultrasound guidance. Ultrasound evaluation was performed by experienced rheumatologists. Ultrasound scans were performed using a Philips HD15 ultrasound system (Philips, Amsterdam, Netherlands), employing 12 to 5 $\mathrm{MHz}$ and 15 to $7 \mathrm{MHz}$ transducers. Gray scale (GS) and power Doppler (PD) were graded using a semi-quantitative score (o to 3) [17]. A composite score for synovitis according to the Outcome Measures in Rheumatology scoring system was recorded in the ultrasound scan report [18]. The steroid used for injection at this hospital is triamcinolone hexacetonide, and the dose was decided at the discretion of the treating rheumatologist.

\section{Statistical analysis}

When selecting IAGI-naive patients, age and sex were used as matching factors. The age groups were $<50$ years, 50 to 59 years, 60 to 69 years, and $\geq 70$ years. When 
combining age group and sex, an IAGI-naive patient was randomly selected for each IAGI patient using a random number. Categorical variables were expressed as numbers and percentages, and continuous variables as the median and IQR. A two-tailed Mann-Whitney U test was used for group comparison. For categorical variables, the chi-square or Fisher's exact test were used as appropriate. Conditional logistic regression analysis was used to investigate various predictors, including whether IAGI had been performed or not, for achieving remission or LDA at 3 months and radiographic progression after 2 years. The models were run in a univariate manner, and then variables with $p<0.2$ were tested in a multivariate model. In addition, conventional risk factors that could influence the treatment response and radiographic progression, such as disease duration (early vs. established RA), smoking history, RF, or ACPA, were also considered as potential confounders. The most relevant regression model was selected based on minimization of the Aikake Information Criterion and parsimony. The results of these analyses are presented as odds ratio (OR) with $95 \%$ confidence interval (CI). Statistical analysis of pooled data was performed using SAS version 9.4 (SAS Institute, Cary, NC, USA) and graphs were drawn using GraphPad Prism 8 (GraphPad Software, San Diego, CA, USA).

\section{RESULTS}

\section{Patient characteristics}

Patients with early RA were younger than patients with established RA (50 and 56 years old, respectively; $p=$ $0.027)$. More patients with established RA received IAGI than those with early RA (60\% vs. 40\%, respectively; $p$ $=0.005)$. The baseline characteristics of the IAGI and IAGI-naive groups were well-balanced overall (except for hypertension). More IAGI-treated patients than IAGI-naive patients were hypertensive (Table 1).

\section{Distribution of active arthritis at the hand and wrist}

The wrist joints were most frequently affected (59\%), followed by the PIP (42\%) and MCP joints (26\%). Patients with active arthritis in the PIP joints were less-frequently treated with IAGI (40\% vs. 60\%, $p<0.001$ ). In total, $61 \%$ of patients underwent ultrasound examination. Only a few IAGI-naive patients underwent ultrasound exam- ination $(n=18)$. The wrist joints showed higher GS and PD grades than the MCP and PIP joints ( $p=0.005$ and $p<0.001$, respectively) (Fig. 2A), whereas the PIP joints presented lower GS and PD grades than the wrist and MCP joints ( $p=0.057$ and $p=0.006$, respectively) (Fig. $2 A$ ). GS and PD grades were similar between patients with established RA and early RA (Fig. 2B).

\section{Treatment after onset of active arthritis}

There was no difference in the use of DMARDs after onset of active arthritis between patients treated and patients not treated with IAGI (Table 1). Leflunomide and biologic DMARDs were used more frequently in patients with established RA than in those with early RA (leflunomide, 24\% vs. 18\%, $p=0.061$; biologic DMARDs, $13 \%$ vs. $4 \%, p=0.058)$. Oral glucocorticoids and NSAIDs were used at similar frequencies in IAGI and IAGI-naive patients with early RA. In those with established RA, a higher proportion of patients not treated with IAGI received oral glucocorticoids and NSAIDs (Table 1).

\section{The short-term efficacy of IAGI}

The baseline DAS28 was similar for patients treated and not treated with IAGI. The DAS28 fell after 3 months in both groups (Fig. $3 \mathrm{~A}$ and $3 \mathrm{~B}$ ). In patients with early and established RA, a higher proportion of those receiving IAGI achieved remission or LDA after 3 months (early RA, $67 \%$ and $46 \%, p=0.099$; established RA, $68 \%$ and $48 \%, p=0.014$ ) (Fig. $3 \mathrm{C}$ ).

On the next visit after IAGI, $78 \%$ of patients with early RA showed resolution of swollen joints; the same was true for $70 \%$ of patients with established RA $(p=0.410)$. No adverse events were documented after IAGI. The proportion of patients who received repeated injection into the same joint within 6 months was similar for those with early and established RA ( $14 \%$ and $11 \%$, respectively, $p>0.999$ ).

\section{The long-term efficacy of IAGI}

Plain hand radiographs were followed up at a median of 2.1 years (IQR, 1.5 to 2.9) after onset of active arthritis. Overall, $31 \%$ of patients $(n=73)$ showed radiographic progression $(\Delta \mathrm{HSS} / \mathrm{year} \geq 2)$; most of these were patients with established RA ( $n=62,85 \%)$. Among patients with early RA, $10 \%$ of IAGI-naive and $26 \%$ of IAGI patients showed radiographic progression $(p=0.099)$. Among 


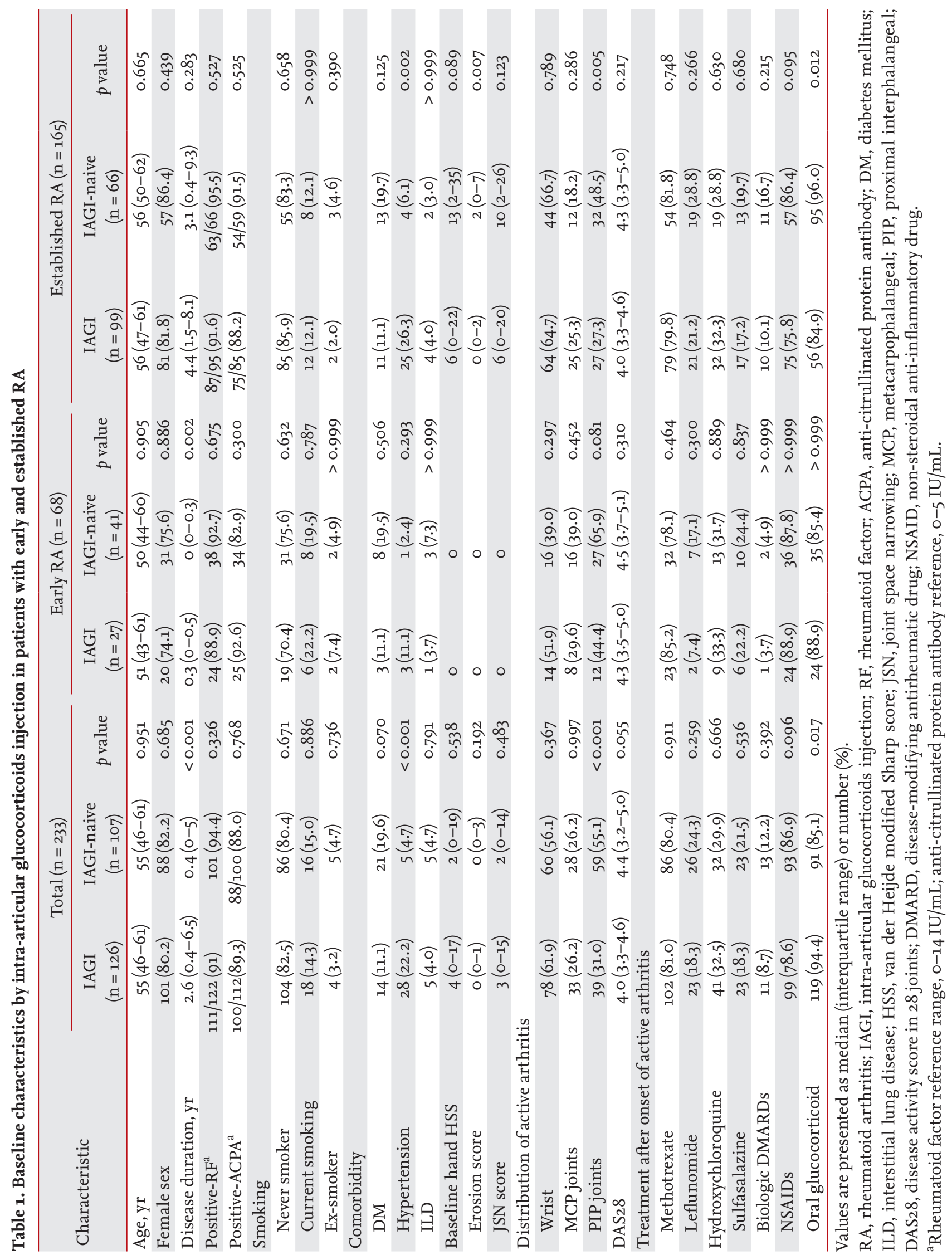



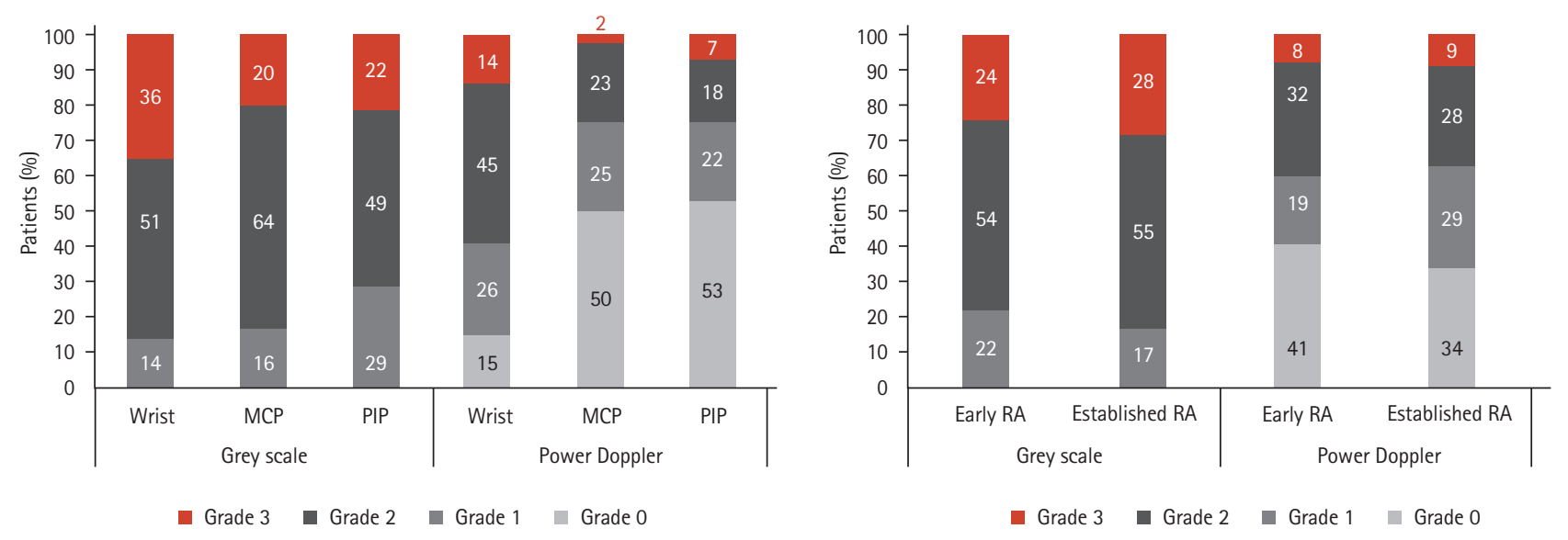

Figure 2. Ultrasound gray scale and power Doppler grades according to joint distribution (A) and early and established RA (B). Data are expressed as percentages. MCP, metacarpophalangeal; PIP, proximal interphalangeal; RA, rheumatoid arthritis.

patients with established RA, the proportion of patients with radiographic progression was not different between patients treated with or without IAGI (37\% of the IAGI group and $38 \%$ of the IAGI-naive group, $p=0.948$ ).

According to joint area, radiographic progression was observed in $42 \%$ of those with active arthritis of the wrist joints, in $23 \%$ of those with active disease in the MCP joints, and in $24 \%$ with active disease in the PIP joints. Radiographic progression after 2 years was greatest in patients with wrist joint arthritis. Radiographic progression after 2 years was associated with higher baseline disease activity indices (Table 2). Patients with higher ultrasound synovitis scores experienced more radiographic progression $(p=0.038)$. When the GS and PD grade were analyzed separately, there was no significant difference between radiographic progressors and non-progressors ( $p=0.103$ and $p=0.612$, respectively) (Table 2 ).

HSS scores at baseline were similar between IAGI and IAGI-naive patients. The median estimated yearly rates for progression in HSS for the hands were o.7 (IQR, o to 3.2) for IAGI patients and o (IQR, o to 2.4) for IAGI-naive patients $(p=0.041)$. This change in HSS was associated with an increase in the JSN score (Fig. 3D). In patients with early RA, the median change in HSS per year was o (IQR, o to 2.1) for the IAGI group; there were no changes in IAGI-naive patients $(p=0.023$ ) (Fig. $3 \mathrm{E}$ ). For those with established RA, the year per year rates of progression were 0.9 (IQR, o to 3.5) for IAGI patients and 1.0 (IQR, o to 3.5) for IAGI-naive patients $(p=0.922)$ (Fig. $3 \mathrm{~F}$ ).

\section{Predictors for achieving remission or LDA after 3 months of active arthritis}

Early RA, wrist joint involvement, and use of oral glucocorticoids were not associated with early remission or LDA. On the other hand, IAGI, use of biologic DMARDs, and baseline DAS28 were identified as independent predictors of achieving remission or LDA after 3 months of active arthritis (Table 3).

\section{Predictors for radiographic progression}

Univariate analysis revealed that medications received after onset of active arthritis were not associated with radiographic progression, nor was the presence of RF or ACPA. In addition, receipt of IAGI was not associated with radiographic progression (Table 3). After adjusting for early RA and baseline HSS, arthritis of the wrist was associated with radiographic progression after a median 2 years. In addition, achieving remission or LDA in 3 months was negatively associated with radiographic progression (Table 3).

In patients who received IAGI, univariate analysis revealed that the ultrasound synovitis score, resolution of joint swelling after IAGI, wrist arthritis, achievement of LDA in 3 months, baseline HSS, and the DAS28 were associated with radiographic progression (Table 3). Multivariate analysis identified a higher baseline HSS, and early achievement of LDA in 3 months as independent predictors of radiographic progression. 

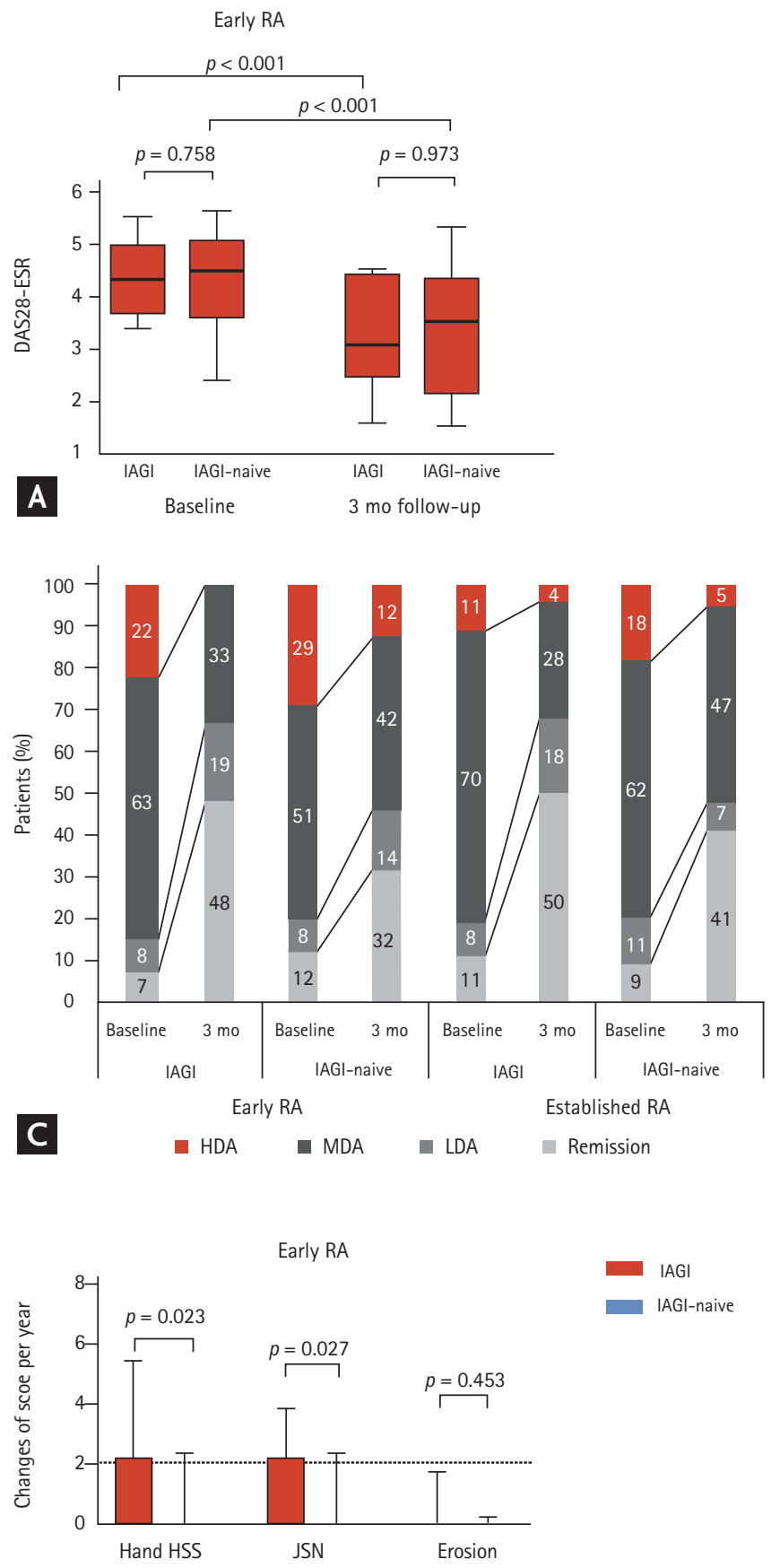

E
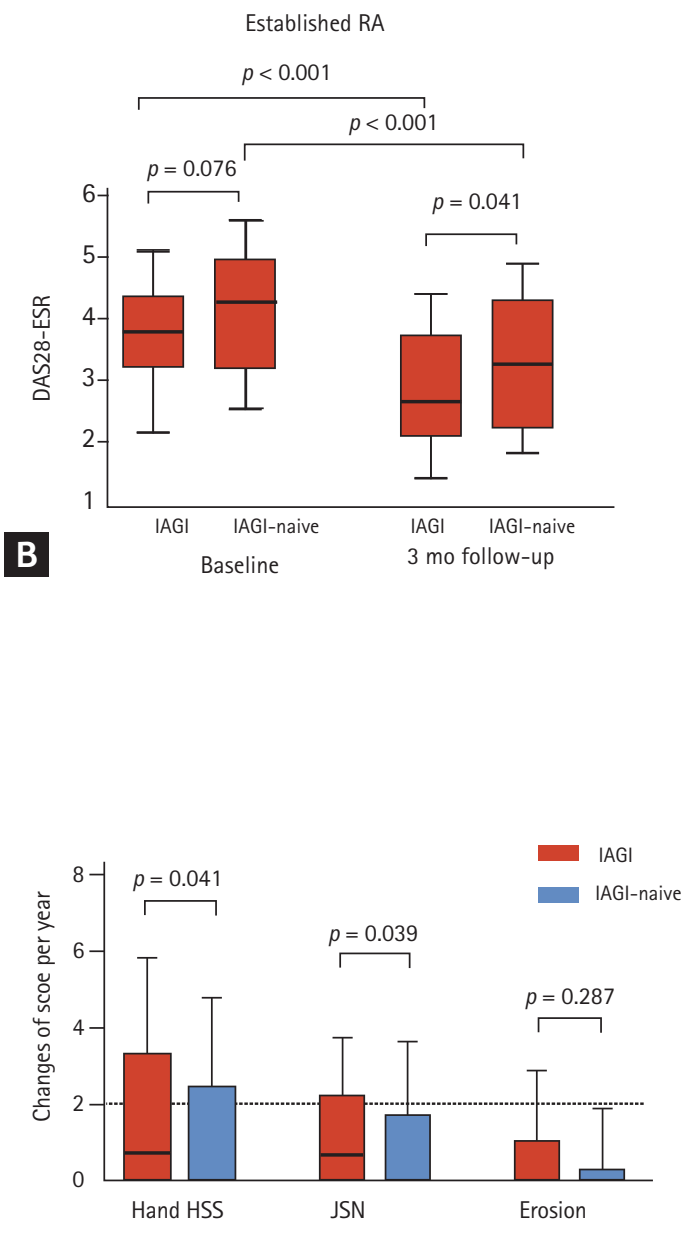

D

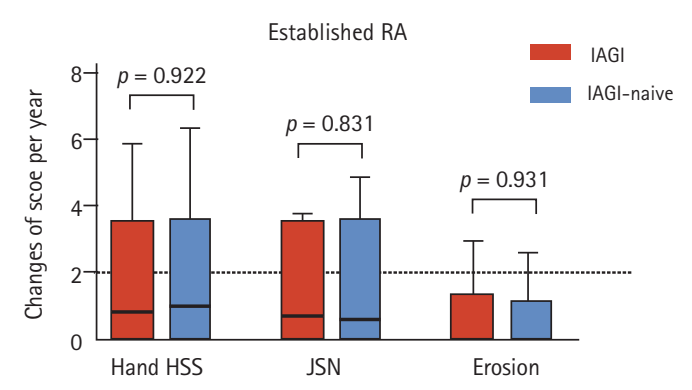

$\mathbf{F}$

Figure 3. (A, B) The changes in the disease activity score in 28 joints (DAS28) of patients with active arthritis over 3 months follow-up. (C) Percentage of patients in different disease activity categories according to the DAS28: DAS28 < 2.6 (remission), $2.6 \leq$ $\mathrm{DAS}_{2} 8 \leq 3.2$ (low disease activity [LDA]), $3.2<\mathrm{DAS} 28 \leq 5.1$ (moderate disease activity [MDA]), and DAS28 $>5.1$ (high disease activity [HDA]). (D) Median changes in the van der Heijde modified Sharp score (HSS) per year in all patients with active arthritis of the wrist or hand joints and treated with (or without) intra-articular glucocorticoids injection (IAGI). (E, F) The rate of annual change in HSS in the IAGI and IAGI-naive groups was analyzed separately for those with early and established rheumatoid arthritis (RA). The boxes define the lower quartile, medium quartile, and upper quartile. The whiskers denote the maximum and minimum values. ESR, erythrocyte sedimetation rate; JSN, joint space narrowing. 
Table 2. Comparison of radiographic progressors and non-progressors

\begin{tabular}{|c|c|c|c|}
\hline Variable & $\begin{array}{l}\text { Radiographic progressors } \\
\qquad(\mathrm{n}=73)\end{array}$ & $\begin{array}{l}\text { Radiographic non-progressors } \\
\qquad(\mathrm{n}=160)\end{array}$ & $p$ value \\
\hline Early RA & $11(15.1)$ & $57(35.6)$ & 0.001 \\
\hline IAGI & $44(60.3)$ & $82(51.3)$ & 0.200 \\
\hline Repeat injection within 6 months & $7 / 44(15 \cdot 9)$ & $10 / 82(12.2)$ & 0.561 \\
\hline \multicolumn{4}{|l|}{ Joint involvement } \\
\hline Wrist joints & $58(79 \cdot 5)$ & $80(50.0)$ & $<0.001$ \\
\hline MCP joints & $14(19.2)$ & $47(29.4)$ & 0.101 \\
\hline PIP joints & $24(32.9)$ & $74(46.3)$ & 0.055 \\
\hline \multicolumn{4}{|l|}{ Baseline disease activities } \\
\hline Swollen joint counts & $2(1-3)$ & $1(1-2)$ & 0.039 \\
\hline Tender joint counts & $2(1-4)$ & $2(1-4)$ & 0.257 \\
\hline $\mathrm{ESR}, \mathrm{mm} / \mathrm{hr}$ & $42(17-69)$ & $27(15-50)$ & 0.009 \\
\hline $\mathrm{CRP}, \mathrm{mg} / \mathrm{dL}$ & $1.1(0.4-2.5)$ & $0.5(0.1-1.2)$ & 0.001 \\
\hline $\mathrm{DAS}_{28}$ & $4.5(3.8-5.0)$ & $4.0(3.2-4.7)$ & 0.001 \\
\hline Ultrasound findings & 44 & 100 & \\
\hline Gray scale & & & $0.103^{\mathrm{a}}$ \\
\hline Grade 1 & $4(9.1)$ & $22(22.0)$ & \\
\hline Grade 2 & $26(59.1)$ & $53(53.0)$ & \\
\hline Grade 3 & $14(31.8)$ & $25(25.0)$ & \\
\hline Power Doppler & & & $0.612^{\mathrm{a}}$ \\
\hline Grade o & $13(29.6)$ & $38(38.0)$ & \\
\hline Grade 1 & $15(34 \cdot 1)$ & $23(23.0)$ & \\
\hline Grade 2 & $11(25.0)$ & $31(31.0)$ & \\
\hline Grade 3 & $5(11.4)$ & $8(8.0)$ & \\
\hline Synovitis score & & & $0.038^{a}$ \\
\hline Grade 1 & $2(4.6)$ & $20(20.0)$ & \\
\hline Grade 2 & $25(56.8)$ & $5^{1}(51.0)$ & \\
\hline Grade 3 & $17(38.6)$ & $29(29.0)$ & \\
\hline
\end{tabular}

Values are presented as number (\%) or median (interquartile range).

RA, rheumatoid arthritis; IAGI, intra-articular glucocorticoids injection; MCP, metacarpophalangeal; PIP, proximal interphalangeal; ESR, erythrocyte sedimentation rate; CRP, C-reactive protein; DAS28, disease activity score in 28 joints.

${ }^{a}$ Calculated using the Mantel-Haenszel chi-square test.

\section{DISCUSSION}

This was a retrospective nested case-control study designed to investigate the short-term and long-term effects of IAGI into the hands or wrist joints as an adjunct to DMARDs in patients with RA. IAGI was a positive predictive factor for achieving remission or LDA at 3 months after the onset of active arthritis, and was not associated with radiographic joint damage in the next
2 years.

The present study demonstrates that joint damage is associated with not achieving remission or LDA in 3 months. In line with the treat-to-target strategy, structural damage in RA is the consequence of inflammation, and its progression is inhibited by controlling inflammation [19]. We found that aggressive interventions such as IAGI or biologic DMARDs were associated with a favorable short-term outcome, although they were 
Table 3. Multivariate logistic regression analysis of data related to achievement of remission or low disease activity at 3 months and radiographic progression after 2 years of active arthritis

\begin{tabular}{|c|c|c|c|c|}
\hline \multirow{2}{*}{ Variable } & \multicolumn{2}{|c|}{ Univariate analysis } & \multicolumn{2}{|c|}{ Multivariate analysis } \\
\hline & OR $(95 \% \mathrm{CI})$ & $p$ value & OR $(95 \% \mathrm{CI})$ & $p$ value \\
\hline \multicolumn{5}{|l|}{ Achieving remission or LDA in 3 months } \\
\hline Early RA (vs. established RA) & $0.796(0.450-1.407)$ & 0.432 & $1.214(0.553-2.663)$ & 0.629 \\
\hline Current smoker (vs. non-smoker) & $1.864(0.847-4.104)$ & 0.122 & $2.069(0.756-5.661)$ & 0.157 \\
\hline IAGI (vs. without IAGI) & $2.276(1.338-3.874)$ & 0.003 & $2.324(1.174-4.600)$ & 0.016 \\
\hline Baseline DAS28, /score & $0.227(0.150-0.342)$ & $<0.001$ & $0.224(0.144-0.347)$ & $<0.001$ \\
\hline Wrist involvement & $0.571(0.332-0.980)$ & 0.042 & $0.861(0.425-1.745)$ & 0.679 \\
\hline Biologic DMARDs & $3.916(1.555-9.858)$ & 0.004 & $3.971(1.160-13.591)$ & 0.028 \\
\hline MTX & $0.729(0.371-1.432)$ & 0.359 & & \\
\hline LEF & $0.685(0.364-1.290)$ & 0.242 & & \\
\hline Oral glucocorticoids & $0.583(0.230-1.477)$ & 0.256 & & \\
\hline \multicolumn{5}{|l|}{ Radiographic progression } \\
\hline Early RA (vs. established RA) & $0.321(0.156-0.658)$ & 0.001 & $0.526(0.221-1.254)$ & 0.140 \\
\hline Current smoker (vs. non-smoker) & $0.522(0.216-1.262)$ & 0.145 & $0.690(0.258-1.851)$ & 0.461 \\
\hline RF positive (vs. negative) & $0.618\left(0.225^{-1.695)}\right.$ & 0.347 & & \\
\hline ACPA positive (vs. negative) & $0.938(0.380-2.311)$ & 0.889 & & \\
\hline Baseline DAS28, /score & $1.619(1.232-2.129)$ & 0.001 & $1.400(0.979-2.001)$ & 0.065 \\
\hline Wrist involvement & $3.867(2.025-7.383)$ & $<0.001$ & $2.569(1.256-5.253)$ & 0.010 \\
\hline LDA in 3 months (vs. MDA/HDA) & $0.355(0.201-0.628)$ & $<0.001$ & $0.456(0.220-0.945)$ & 0.035 \\
\hline Baseline hand HSS, /score & $1.032(1.016-1.048)$ & $<0.001$ & $1.023(1.005-1.041)$ & 0.011 \\
\hline IAGI (vs. without IAGI) & $1.443(0.823-2.532)$ & 0.201 & $1.916(0.976-3.762)$ & 0.058 \\
\hline \multicolumn{5}{|c|}{ Radiographic progression among patients treated with IAGI } \\
\hline Early RA (vs. established RA) & $0.596(0.238-1.491)$ & 0.271 & $1.089(0.340-3.489)$ & 0.886 \\
\hline Wrist joint involvement & $2.308(1.061-5.018)$ & 0.003 & $2.665(0.972-7.305)$ & 0.057 \\
\hline Baseline DAS28, /score & $1.802(1.216-2.670)$ & 0.003 & $1.468(0.904-2.384)$ & 0.121 \\
\hline Resolution of joint swelling after IAGI & $2.262(1.110-5.460)$ & 0.025 & & \\
\hline Repeated injection (vs. single injection) & $1.362(0.480-3.870)$ & 0.561 & & \\
\hline LDA in 3 months (vs. MDA/HDA) & $0.345(0.160-0.745)$ & 0.008 & $0.364(0.144-0.916)$ & 0.032 \\
\hline Baseline hand SHS, /score & $1.036(1.012-1.061)$ & 0.003 & $1.034(1.007-1.061)$ & 0.013 \\
\hline Synovitis score, /grade & $1.800(1.028-3.154)$ & 0.040 & $1.014(0.488-2.107)$ & 0.971 \\
\hline
\end{tabular}

OR, odds ratio; CI, confidence interval; LDA, low disease activity; RA, rheumatoid arthritis; IAGI, intra-articular glucocorticoid injection; DAS28, disease activity score in 28 joints; DMARD, disease-modifying antirheumatic drug; MTX, methotrexate; LEF, leflunomide; RF, rheumatoid factor; ACPA, anti-citrullinated protein antibody; MDA, moderate disease activity; HDA, high disease activity; HSS, van der Heijde modified Sharp score.

not associated directly with radiographic progression. In addition, patients with higher ultrasound synovitis scores or higher disease activity indices showed greater radiographic progression in the next 2 years, although this result was not significant after adjusting for early achievement of LDA. This suggests that how one controls active arthritis is more important than the severity of arthritis.

Both erosions and JSN are aspects of structural damage in RA. Semi-quantitative scoring of erosions is rep- 
resentative of structural damage to bone, whereas JSN scores are a surrogate for loss of cartilage [20]. From this viewpoint, JSN rather than erosion accounted for radiographic changes in patients receiving IAGI, particularly in patients with early RA. IAGI reduces progression of erosions by regulating inflammation, as well as by down-modulating expression of bone-destructive markers [5]. At the same time, IAGI accelerates progression of JSN by exerting detrimental effects on cartilage, including chondrotoxicity and breakdown of the extracellular matrix [21-23]. In the present study, we found that the degree of JSN progression was very low, and appeared only in early RA patients treated with IAGI. On subgroup analysis of the radiographic progressors who had been treated with IAGI, early RA patients showed a worse response at 3 months after IAGI than established RA patients (data not shown). Although the number of early RA patients with radiographic progression was small, these results may suggest that early RA patients with no treatment response after 3 months should be considered for more aggressive treatment.

Wrist joints are vulnerable to damage; indeed, they are affected in up to $50 \%$ of patients with RA within the first 2 years after diagnosis, increasing to more than $90 \%$ after 10 years $[24,25]$. The wrist is a complex joint and the radiocarpal, intercarpal, midcarpal, carpometacarpal, and intermetacarpal joints often intercommunicate through a common synovial cavity [26]. As the synovium is the principal target of inflammation in RA, proliferating fibroblast-like synoviocytes and proinflammatory cytokines may spread through the common synovial cavity and alter joint physiology over time. Indeed, we found that the wrist joint showed a higher GS and PD grade than the PIP or MCP joints, as well as being associated with radiographic progression after 2 years. This result suggests that more aggressive interventions should be considered in those with active arthritis of the wrist joints.

This study has several potential limitations. First, we did not rule out the possibility of selection bias between IAGI and IAGI-naive patients. Because ultrasound information was available mostly for the IAGI group, we did not compare the degree of synovitis between the two groups. Patients treated with IAGI may have had more severe arthritis and were therefore selected for injection by the rheumatologist. This may have resulted in an association between IAGI and structural damage in patients with early RA. In addition, the number of patients with early RA who received IAGI was too small to provide validated evidence regarding radiographic progression. Second, as the dose of intra-articular injected triamcinolone was not unified, we could not analyze the cumulative dose, which may be associated with radiographic progression. Third, the treatment regimen for each patient changed over the 2 years; we did not investigate the effects of all medications on joint damage. Fourth, this study was based on semi-quantitative scoring, which may be influenced by the positioning of the joints during radiography and by joint swelling [20]. Thus, further studies that measure cartilage area before and after IAGI are needed to confirm the actual effects of IAGI on patients with RA. Fifth, short-term follow-up ultrasound examinations were not performed after IAGI, and changes in the injected joint were not evaluated by ultrasound examination. The treatment response to IAGI may vary depending on the accuracy of intra-articular placement of the glucocorticoid, degree of synovitis, and degree of joint use [2,27,28]. Although physical examinations were used to evaluate qualitative changes in the swollen joint after IAGI, we did not evaluate semi-quantitative changes that could be estimated by ultrasound examinations. Additionally, the ultrasound synovitis score was estimated and recorded by a single examiner in a real-world outpatient setting. It was not validated by two or more independent examiners.

To the best of our knowledge, the literature on the long-term efficacy of IAGI with respect to radiographic changes is very limited. Previous studies that report the long-term effects of IAGI focused on suppression of inflammation $[2,29]$. Unlike previous studies that included only patients with early RA [2-4,6,30], the present study includes patients with early and established RA, which will provide real-world data about the effects of IAGI.

In conclusion, IAGI given to patients with RA as an adjunct to DMARDs therapy may rapidly reduce the severity of arthritis without causing significant longterm structural damage. Our results emphasize the importance of suppressing inflammation in patients with RA. IAGI can be considered as an adjunctive therapy for treat-to-target strategies. Wrist arthritis showed greater potential for radiographic progression; therefore, arthritis in this joint should be treated more aggressively. 


\section{KEY MESSAGE}

1. Intra-articular glucocorticoid injection (IAGI), as an adjunctive to disease-modifying antirheumatic drugs treatment, reduced disease activity rapidly. However, some patients receiving IAGIs showed more joint space narrowing after 2 years, although it was not significant, after adjusting for treatment and baseline disease activity.

2. Early achievement of low disease activity was an important factor associated with radiographic joint damage.

3. The wrist is more vulnerable to joint damage and requires more aggressive treatment.

\section{Conflict of interest}

No potential conflict of interest relevant to this article was reported.

\section{Acknowledgments}

This work was supported by a grant from the National Research foundation of Korea (NRF-2018R1D1A1Bo7045491).

\section{REFERENCES}

1. Smolen JS, Landewe R, Bijlsma J, et al. EULAR recommendations for the management of rheumatoid arthritis with synthetic and biological disease-modifying antirheumatic drugs: 2016 update. Ann Rheum Dis 2017;76:960-977.

2. Hetland ML, Ostergaard M, Ejbjerg B, et al. Short- and long-term efficacy of intra-articular injections with betamethasone as part of a treat-to-target strategy in early rheumatoid arthritis: impact of joint area, repeated injections, MRI findings, anti-CCP, IgM-RF and CRP. Ann Rheum Dis 2012;71:851-856.

3. Haugeberg G, Morton S, Emery P, Conaghan PG. Effect of intra-articular corticosteroid injections and inflammation on periarticular and generalised bone loss in early rheumatoid arthritis. Ann Rheum Dis 2011;70:184-187.

4. Hetland ML, Stengaard-Pedersen K, Junker P, et al. Aggressive combination therapy with intra-articular gluco- corticoid injections and conventional disease-modifying anti-rheumatic drugs in early rheumatoid arthritis: second-year clinical and radiographic results from the CIMESTRA study. Ann Rheum Dis 2008;67:815-822.

5. Makrygiannakis D, af Klint E, Catrina SB, et al. Intraarticular corticosteroids decrease synovial RANKL expression in inflammatory arthritis. Arthritis Rheum 2006;54:14631472.

6. Kuusalo LA, Puolakka KT, Kautiainen H, et al. Intra-articular glucocorticoid injections should not be neglected in the remission targeted treatment of early rheumatoid arthritis: a post hoc analysis from the NEO-RACo trial. Clin Exp Rheumatol 2016;34:1038-1044.

7. Menon N, Kothari SY, Gogna A, Sharma R. Comparison of intra-articular glucocorticoid injections with DMARDs versus DMARDs alone in rheumatoid arthritis. J Assoc Physicians India 2014;62:673-676.

8. Terslev L, Torp-Pedersen S, Qvistgaard E, Danneskiold-Samsoe B, Bliddal H. Estimation of inflammation by Doppler ultrasound: quantitative changes after intra-articular treatment in rheumatoid arthritis. Ann Rheum Dis 2003;62:1049-1053.

9. McAlindon TE, LaValley MP, Harvey WF, et al. Effect of intra-articular triamcinolone vs saline on knee cartilage volume and pain in patients with knee osteoarthritis: a randomized clinical trial. JAMA 2017;317:1967-1975.

10. Zeng C, Lane NE, Hunter DJ, et al. Intra-articular corticosteroids and the risk of knee osteoarthritis progression: results from the Osteoarthritis Initiative. Osteoarthritis Cartilage 2019;27:855-862.

11. Boesen M, Boesen L, Jensen KE, et al. Clinical outcome and imaging changes after intraarticular (IA) application of etanercept or methylprednisolone in rheumatoid arthritis: magnetic resonance imaging and ultrasound-Doppler show no effect of IA injections in the wrist after 4 weeks. J Rheumatol 2008;35:584-591.

12. Wernecke C, Braun HJ, Dragoo JL. The effect of intra-articular corticosteroids on articular cartilage: a systematic review. Orthop J Sports Med 2015;3:2325967115581163.

13. Aletaha D, Neogi T, Silman AJ, et al. 2010 Rheumatoid arthritis classification criteria: an American College of Rheumatology/European League Against Rheumatism collaborative initiative. Arthritis Rheum 2010;62:25692581.

14. Van der Heijde DM. Plain X-rays in rheumatoid arthritis: overview of scoring methods, their reliability and appli- 
cability. Baillieres Clin Rheumatol 1996;10:435-453.

15. Anderson J, Caplan L, Yazdany J, et al. Rheumatoid arthritis disease activity measures: American College of Rheumatology recommendations for use in clinical practice. Arthritis Care Res (Hoboken) 2012;64:640-647.

16. Bruynesteyn K, van der Heijde D, Boers M, et al. Determination of the minimal clinically important difference in rheumatoid arthritis joint damage of the Sharp/van der Heijde and Larsen/Scott scoring methods by clinical experts and comparison with the smallest detectable difference. Arthritis Rheum 2002;46:913-920.

17. Szkudlarek M, Court-Payen M, Jacobsen S, Klarlund M, Thomsen HS, Ostergaard M. Interobserver agreement in ultrasonography of the finger and toe joints in rheumatoid arthritis. Arthritis Rheum 2003;48:955-962.

18. Forien M, Ottaviani S. Ultrasound and follow-up of rheumatoid arthritis. Joint Bone Spine 2017;84:531-536.

19. Grigor C, Capell H, Stirling A, et al. Effect of a treatment strategy of tight control for rheumatoid arthritis (the TICORA study): a single-blind randomised controlled trial. Lancet 2004;364:263-269.

20. Van der Heijde D. Erosions versus joint space narrowing in rheumatoid arthritis: what do we know? Ann Rheum Dis 2011;70 Suppl 1:i116-i118.

21. Beitzel K, McCarthy MB, Cote MP, et al. The effect of ketorolac tromethamine, methylprednisolone, and platelet-rich plasma on human chondrocyte and tenocyte viability. Arthroscopy 2013;29:1164-1174.

22. Fubini SL, Todhunter RJ, Burton-Wurster N, Vernier-Singer M, MacLeod JN. Corticosteroids alter the differentiated phenotype of articular chondrocytes. J Orthop
Res 2001;19:688-695.

23. Shen C, Cai GQ, Peng JP, Chen XD. Autophagy protects chondrocytes from glucocorticoids-induced apoptosis via $\mathrm{ROS} / \mathrm{Akt} / \mathrm{FOXO}_{3}$ signaling. Osteoarthritis Cartilage 2015;23:2279-2287.

24. Terao C, Hashimoto M, Yamamoto K, et al. Three groups in the 28 joints for rheumatoid arthritis synovitis: analysis using more than 17,000 assessments in the KURAMA database. PLoS One 2013;8:e59341.

25. Kanazawa T, Nishino J, Tohma S, Tanaka S. Analysis of the affected joints in rheumatoid arthritis patients in a large Japanese cohort. Mod Rheumatol 2013;23:44-49.

26. Kijima Y, Viegas SF. Wrist anatomy and biomechanics. J Hand Surg Am 2009;34:1555-1563.

27. Naredo E, Cabero F, Beneyto P, et al. A randomized comparative study of short term response to blind injection versus sonographic-guided injection of local corticosteroids in patients with painful shoulder. J Rheumatol 2004;31:308-314.

28. Weitoft T, Ronnblom L. Randomised controlled study of postinjection immobilisation after intra-articular glucocorticoid treatment for wrist synovitis. Ann Rheum Dis 2003;62:1013-1015.

29. Gvozdenovic E, Dirven L, van den Broek M, et al. Intra articular injection with corticosteroids in patients with recent onset rheumatoid arthritis: subanalyses from the BeSt study. Clin Rheumatol 2014;33:263-267.

30. Furtado RN, Oliveira LM, Natour J. Polyarticular corticosteroid injection versus systemic administration in treatment of rheumatoid arthritis patients: a randomized controlled study. J Rheumatol 2005;32:1691-1698. 